\title{
Vulvar Contact Dermatitis: Effect of a Treatment with a Anti-Inflammatory, Moisturizing and Healing Product on the Course of the Disease
}

\author{
Filippo Murina*, Franco Vicariotto \\ Department of Vulvar Disease, V. Buzzi Hospital, University of Milan, Milan, Italy \\ Email: "filippomurina@tin.it
}

Received 9 January 2016; accepted 21 February 2016; published 24 February 2016

Copyright (C) 2016 by authors and Scientific Research Publishing Inc.

This work is licensed under the Creative Commons Attribution International License (CC BY). http://creativecommons.org/licenses/by/4.0/

(c) (i) Open Access

\begin{abstract}
Background: Vulvar contact dermatitis is inflammatory disorders of genital skin causing itch, burning, and plaques with overlying excoriation and linear fissures. Objective: To assess the effectiveness of a soft foam based on Tiab ${ }^{\circledR}$ system (TF), complex of titanium micro crystals covalently bound with silver ions associated with hyaluronic acid, as a treatment of vulvar contact dermatitis. Method: 36 patients with the diagnosis of vulvar contact dermatitis received an 10-days treatment, during which the subjects applied TF, complex of titanium micro crystals covalently bound with silver ions associated with hyaluronic acid on the affected vulvar surfaces, used once a day. Clinical symptoms (itching and burning) and signs (vulvar surface disruption) were documented at baseline and after 1 months of follow-up. Results: There was a significant reduction of symptoms between the basal scores and the symptoms complaints 1 months of follow-up. Moreover a significant reduction of excoriations and linear fissures was observed. Conclusion: The present study shows that application of TF can be an effective agents for the reduction of symptom and sign of vulvar contact dermatitis, and the medical device can be used as a preventive agent in predisposing condition of vulvar discomfort.
\end{abstract}

\section{Keywords}

Vulvar Contact Dermatitis, Moisturizer, Vulvar Itch, Vulvar Discomfort

\section{Introduction}

Vulvar disease is often multifactorial, and contact dermatitis of the vulva is frequently an important contributor.

${ }^{*}$ Corresponding author.

How to cite this paper: Murina, F. and Vicariotto, F. (2016) Vulvar Contact Dermatitis: Effect of a Treatment with a Anti-Inflammatory, Moisturizing and Healing Product on the Course of the Disease. Open Journal of Obstetrics and Gynecology, 6, 117-121. http://dx.doi.org/10.4236/ojog.2016.62014 
Although a variety of agents have been implicated in vulvar dermatitis, the susceptibility of this tissue to inflammation caused by topical and micro traumatic agents may be underestimated [1]. Contact dermatitis is an inflammation of the skin resulting from an external agent that acts as an irritant. Although it is not usually the primary cause of vulvar symptoms, it is often the compounding factor in patients complaining of persistent vulvar pruritus, irritation, or burning. The characteristic symptoms, such as vulvar itching and soreness will frequently adversely affect the quality of life of affected women. Typically, there is a presentation of varying redness, swelling, and scaling. There may be erosions or frank ulcers with marked erythema and swelling.

The reported incidence of contact dermatitis in the anogenital area varies. From the vulvar clinic in Oxford, UK, $20 \%$ - 30\% of cases had dermatitis, and of these, $26 \%$ had relevant patch tests [2].

The increased tendency to irritant dermatitis may cause problems by itself, or act as a risk factor for further development of others vulvar dermatoses.

Common irritants include soaps, antiseptics, lubricants, spermicides, tampons, sanitary pads, and synthetic underwear. Irritant contact dermatitis may be due to poor hygiene habits or excess use of soap or prolonged wearing of wet swimming suits [3].

The clinical alterations of vulvar contact dermatitis are responsible for changes in vulvar skin barrier function that enhance the symptoms (dehydration and impaired lipid skin film).

The barrier function of vulvar skin is substantially weaker than at other anatomical sites, and moisture, friction, urine, and vaginal discharge all contribute to vulvar irritation by weakening barrier function. The weak irritants are the more common, often insidious offenders.

Fear of detectable odor or infection may drive overzealous hygiene habits, and many soaps and detergent cleansers are too harsh for the vulva. When one considers the wide spectrum of vulvar contact dermatitis, a common rash derived from irritant diaper contact dermatitis.

These diaper rashes are precipitated by constant moisture, occlusion, and frictional forces.

Excess wetting macerates the stratum corneum so that it is fragile and friction causes further trauma. Clinically, the patients complain of varying degrees of itching, burning, and irritation. The onset may be sudden or gradual depending on the etiology. The patient may be aware of the offending product that precipitated the problem or may be totally mystified.

Management of vulvar contact dermatitis consists in the removal of all irritants and potential allergens and application of topical steroids until the skin returns to normal.

Use of emollient soap substitute and topical corticosteroid - the choice of preparation will depend on severity, $1 \%$ Hydrocortisone ointment in milder cases, or betamethasone valerate $0.025 \%$ or clobetasol propionate $0.05 \%$ for limited periods are important elements of care [4].

A combined preparation containing antifungal and/or antibiotic may be required for short-term use (to try and avoid the development of contact allergy) if secondary infection suspected.

The aim to this study was to assess the effectiveness of a soft foam based on Tiab® system (TF), complex of titanium micro crystals covalently bound with silver ions associated with hyaluronic acid, in the treatment of vulvar contact dermatitis.

Our premise is that TF can acts not only as a moisturizing agent, but it may has a pivotal action in resolution of symptoms and promoting wound healing and restoration of the protective film skin altered in patients with vulvar contact dermatitis.

\section{Materials and Methods}

Thirty-six women, older than 18 years, affected by vulvar contact dermatitis were recruited.

For each patient, an evaluation of subjective symptoms and objective genital signs, identified as typical of vulvar contact dermatitis, was recorded before the start of treatment and after 1 months of the study. Both signs and symptoms were evaluated according to an increasing severity score from 0 to 3, in which 0 means absent, 1 means mild, 2 means moderate, and 3 means severe.

All of the patients received an 10-days treatment, during which the subjects applied a soft foam based on Tiab® system (TF), complex of titanium micro crystals covalently bound with silver ions associated with hyaluronic acid on the affected vulvar surfaces, used once a day.

The appearance of vulvar surface regarding the redness, swelling, excoriations and linear fissures if presents, was considered as a secondary outcome. Grading of vulvar appearance was quantified using a four-point scoring 
system $(0=$ no lesions, $1=$ decreased $>50 \% ; 2=$ decreased $<50 \%$ e $3=$ unchanged $)$.

The EPI-INFO version 6.0 (Centers for Disease Control and Prevention, Atlanta, GA) was used for all statistical analyses. Descriptive statistical analyses (means, standard deviations, and percentages) were performed using the Student $t$ test. Differences were considered significant at $\mathrm{p} \geq 0.05$.

\section{Results}

Thirty-six women affected by vulvar contact dermatitis, aged from 22 to 59 years (median age $=41.5 \mathrm{y}$ ), were enrolled in this study. Table 1 shows the prevalence of each symptom and sign in all patients, and the source of dermatitis for the study population.

The symptoms at the first consult were itching and burning in 31 (86\%), 29 (80.5\%) women respectively. In terms of the subjective outcome, as reported by the patients (itching and burning) there was a significant reduction of symptoms between the basal scores and the symptoms complaints at 1 months follow-up (Figure 1). Moreover we observed a significant reduction of vulvar appearance after the treatment, and fissures and erosions were the two clinical signs that showed most improvement across both groups of patients. All patients confirmed that they had applied the foam. During the entire study no drop out related to the topical treatments was observed; only three patients experienced a mild transient burning after TF application.

\section{Discussion}

Contact dermatitis is a primary contributor to vulvar discomfort and is the chief reason why women seek medical intervention. The present study shows that daily application of TF can be effective for the treatment of vulvar contact dermatitis.

Table 1. Personal and baseline characteristics of the subjects.

\begin{tabular}{lc}
\multicolumn{1}{c}{ Variable } & Data \\
\hline Duration of symptoms (days) & $7.4(5.22)$ \\
Diagnosis (numbers) & Total n. 36 \\
- Post intimate soaps & 9 \\
- Post lubricants & 7 \\
- Post panty liners and/or sanitary pads & 9 \\
- Post topical medicaments & 11 \\
Itching* & $2.90(0.83)$ \\
Burning $^{*}$ & $2.75(0.85)$ \\
\hline
\end{tabular}

Data are presented as mean value \pm standard deviation. ${ }^{*}$ Score $0-3(0=$ none, $1=$ mild, $2=$ moderate and 3 = severe).

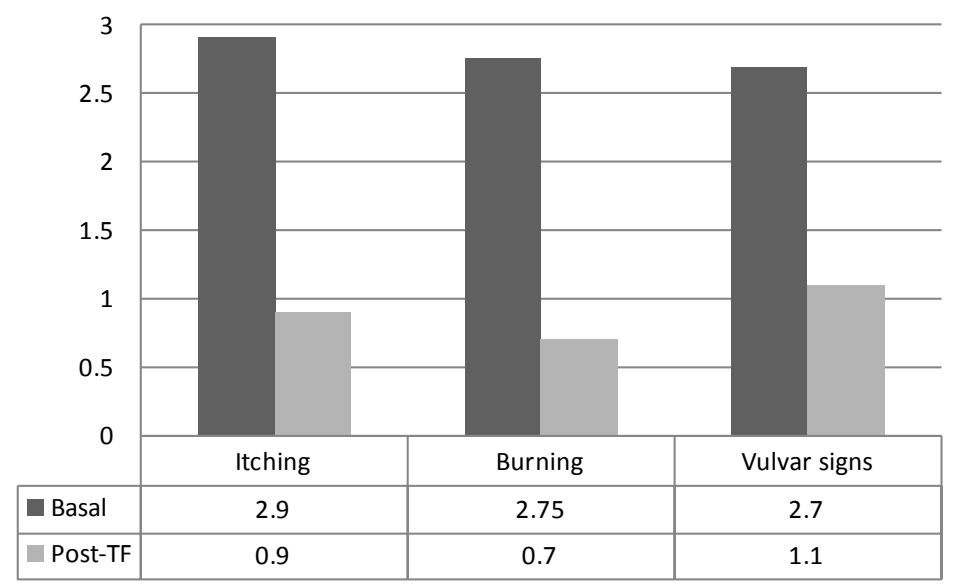

Figure 1. Symptoms after treatment. Score $0-3(0=$ none, $1=$ mild, $2=$ moderate and $3=$ severe), means $\mathrm{P}$ value: itching $=0.001$, burning $=0.05$, vulvar signs $=0.03$. 
Vulvar tissue exhibits distinct characteristics in structure, hydration, friction, and occlusion that affect its susceptibility to inflammation. While not widely recognized, these factors may heighten susceptibility to contact irritants and allergens and contribute to making dermatitis the most common vulvar complaint. These distinctions may contribute to heightened vulvar susceptibility to topical irritants and allergens.

Many products can cause irritation of the vulva as well as inadequate vulvar care measures or micro-traumatic factors may play a relevant role. A detailed history should explore whether the patient has adopted any new cleansing routines, has been using new personal care products, douches, perfumes, undergarments and topical medications. Excessive cleansing of the vulvar skin may precipitate to an irritant dermatitis [5].

Many soaps and detergent cleansers are too harsh for the vulva, and patients do not realize the damage that they are doing.

Overzealous hygiene can be a major problem since a cleanser or soap may be followed by powder and, later in the day, by antiseptic wipes.

The use of panty liners has become a common habit among women seeking protection against menstrual blood stains on their clothing, non-menstrual vaginal discharge, and the release of genital odors at social events. Some studies have indicated that the use of tampons or pads interferes with local vulvar and/or vaginal homeostasis mechanisms, and they may provoke a contact dermatitis [6].

Several researchers who have studied the use of panty liners argue that prolonged occlusion of the genital area can change the temperature, $\mathrm{pH}$, and microflora of the vaginal epithelium and vulvar skin, possibly leading to a higher occurrence of contact dermatitis and infections [7].

Urinary incontinence is a very common problem in women, starting in their fifties or even earlier depending on the integrity of their pelvic floor muscles. Pads are worn, and are often inadequate to assure dryness and the patients hide this problem from their healthcare providers because of embarrassment. These women may use normally napkins for incontinence, and pantyhose and/or girdles, can produce the typical glazed vulvar erythema seen in "diaper dermatitis" in infants.

It is important to note that all of the factors of vulvar contact dermatitis above described were indication of use of TF in our study. The diagnosis of vulvar contact dermatitis is made on history and clinical pattern. The history must be thorough since patients often do not recognize the association between their problem and the contactant. Review their routines for hygiene, and for personal care during menstrual periods and with coitus. Ask specific questions as to their use of soaps, cleansers, washcloths, detergents, lubricants, and anti-yeast treatments, for example. Be sure to check all prescription and over-the-counter products. A careful history is the basis for proper approach to disease.

Initial treatment regimen of vulvar contact dermatitis were individualized, with the target outcome being an objective to resolve the vulvar discomfort and to return of the vulvar skin to normal color and texture. Topical corticosteroids represent an appropriate agents for the reduction of inflammation. Triamcinolone $0.1 \%$ ointment bid can be used for moderately irritated dermatitis. If severe, a super-potent topical steroid like clobetasol or halobetasol $0.05 \%$ ointment is prescribed bid for 1 - 3 weeks. The stronger steroids can then be replaced by milder $1 \%-2.5 \%$ hydrocortisone ointment as needed. However, topical steroids are capable to causing local adverse effects, like epidermal atrophy and hypersensitivity reactions [4].

The additional application of a moisturizer seemed to be very important for decreasing symptoms in patients affected by vulvar contact dermatitis. The barrier function of vulvar skin is substantially weaker than at other anatomical sites; moisture, friction, urine, and vaginal discharge all contribute to vulvar irritation by weakening barrier. These factors can be exacerbates in patients with vulvar dermatosis where the integrity of vulvar barrier is damaged. Secondary infection may be manifested by pustules, crusting, and fissuring.

In our study we observed that the a soft foam based on Tiab ${ }^{\circledR}$ system had not only a moisturizer action, it accelerates normalization of the hydrolipidic film, a natural protective barrier for the skin that helps retain moisture and maintain the skin's natural suppleness, and it may also be associated with a reduction in epithelial disruption in the form of excoriations and fissures.

Moreover, the foam based on Tiab ${ }^{\circledR}$ system has a reservoir effect for lengthy maintenance of active ingredient in vulvar surface. This involves a prolonged efficacy of the product and the silver ions are able to exert an anti-inflammatory action on vulvar surface. The innovative patented formula has a high bactericide power combined with a healing action.

Improvement of barrier layer function leads to the restoration of a physiological environment for the nerve endings and it can be accomplished most quickly through the use of soaks. 
In fact the foam formulation creates a protective barrier and it promotes normal scarring and healing through the creation of a favorable microenvironment to the cellular tissue repair [8].

The new foam exists as a liquid pressurized in an aluminum can with a hydrocarbon propellant (propane/ butane), which upon valve actuation forms a foam lattice Patients find foams less dense and therefore generally easier to apply to and spread on the skin surface.

Our important results with the use of the foam based on Tiab ${ }^{\circledR}$ system opens new scenarios in the use of the product. The preventive use of the foam can be used vulvar discomfort if not blocked in the early phase can lead to real pathological changes, or it may be used as a preventive treatment.

Vulnerable conditions like post menstrual use of panty liners, sports practices with stimulation of the vulvar region (e.g. Exercise bike, spinning, riding, etc.) and post-coital irritation can be effectively treated with the foam based on $T i a b{ }^{\circledR}$ system, reducing the risk of evolution in contact dermatitis.

\section{Conclusion}

Foam based on Tiab ${ }^{\circledR}$ system represent an appropriate agents for the reduction of symptom and sign of vulvar contact dermatitis, and the medical device can be used as a preventive agent in predisposing condition of vulvar discomfort.

\section{References}

[1] O’Gorman, S.M. and Torgerson, R.R. (2013) Allergic Contact Dermatitis of the Vulva. Dermatitis, 24, 64-72. http://dx.doi.org/10.1097/DER.0b013e318284da33

[2] Crone, A.M., Stewart, E.J., Wojnarowska, F. and Powell, S.M. (2000) Aetiological Factors in Vulvar Dermatitis. Journal of the European Academy of Dermatology and Venereology, 14, 181-186. http://dx.doi.org/10.1046/j.1468-3083.2000.00080.x

[3] Moyal-Barracco, M. and Wendling, J. (2014) Vulvar Dermatosis. Best Practice \& Research Clinical Obstetrics and Gynaecology, 28, 946-958. http://dx.doi.org/10.1016/j.bpobgyn.2014.07.005

[4] Edwards, S., Bates, C., Lewis, F., Sethi, G. and Grover, D. (2014) 2014 UK National Guideline on the Management of Vulval Conditions. International Journal of Std \& Aids, 8 October 2014.

[5] Lynch, P.J. (2004) Lichen Simplex Chronicus (Atopic/Neurodermatitis) of the Anogenital Region. Dermatology and Therapy, 17, 8-19. http://dx.doi.org/10.1111/j.1396-0296.2004.04002.x

[6] Runeman, B., Rybo, G., Larkö, O. and Faergemann, J. (2003) The Vulva Skin Microclimate: Influence of Panty Liners on Temperature, Humidity and pH. Acta Dermato-Venereologica, 83, 88-92. http://dx.doi.org/10.1080/00015550310007409

[7] Runeman, B., Rybo, G., Forsgren-Brusk, U., Larkö, O., Larsson, P. and Faergemann, J. (2004) The Vulvar Skin Microenvironment: Influence of Different Panty Liners on Temperature, $\mathrm{pH}$ and Microflora. Acta Dermato-Venereologica, 84, 277-284.

[8] Huang, X., Tanojo, H., Lenn, J., Deng, H. and Krochmal, L. (2005) A Novel Foam Vehicle for Delivery of Topical Corticosteroids. Journal of the American Academy of Dermatology, 53, S26-S38. http://dx.doi.org/10.1016/j.jaad.2005.04.028 